\title{
Determinación del consumo de agua potable durante la construcción de viviendas en una zona semi desértica de Chile
}

\section{Determination of drinking water consumption during the construction of houses in a semi-desert area of Chile}

\author{
Amin Nazer ${ }^{1,2}$, Osvaldo Pavez ${ }^{2,3}$, \\ Boris Zúñiga ${ }^{1}$, Luis González ${ }^{1}$
}

\author{
${ }^{1}$ Departamento de Construcción - Universidad de Atacama, Atacama, Copiapó, Chile. \\ e-mail: amin.nazer@uda.cl \\ ${ }^{2}$ Centro Regional de Investigación y Desarrollo Sustentable de Atacama, Universidad de Atacama, Atacama, Copiapó, \\ Chile \\ ${ }^{3}$ Departamento de Ingeniería en Metalurgia - Universidad de Atacama, Atacama, Copiapó, Chile \\ e-mail: osvaldo.pavez@uda.cl; boris.zuniga@alumnos.uda.cl; luis.gonzalez@uda.cl
}

\section{RESUMEN}

En los últimos años, la falta de agua en el norte de Chile se ha incrementado notoriamente, esto ha llevado a una sobreexplotación de los acuíferos subterráneos. En el sector de la construcción, el agua se utiliza en grandes cantidades en diferentes actividades propias del proyecto, por lo que resulta fundamental su cuantificación con el propósito de conocer la demanda real de agua, y de esta forma tener una visión clara y precisa sobre los volúmenes de agua que están siendo usados en diferentes partidas de una obra. En el presente estudio, la determinación de los coeficientes de consumo de agua se efectúa a partir de una serie de datos obtenidos en la construcción de 219 viviendas y 2 salas de multiuso. Para ello, se compararon los resultados teóricos de la demanda de agua, a través de cálculos del proyecto, con respecto al consumo real de agua in situ. A partir de la determinación del consumo teórico y real, los resultados mostraron que la cantidad teórica de agua que debía ser utilizada era de $10.946 \mathrm{~m}^{3}$, bastante diferente de la cantidad real de agua utilizada que fueron $16.283 \mathrm{~m}^{3}$. Así, en la obra se utilizaron $5.337 \mathrm{~m}^{3}$ adicionales, lo que representa un aumento en el consumo de agua con respecto al teórico del orden del 48,8 \%. Las principales actividades consumidoras de agua fueron las siguientes: compactación del suelo, riego para control de partículas en suspensión, curado de hormigones y morteros, campamento de trabajadores, fabricación de hormigones y morteros. Dentro de estas actividades, la que presentó un consumo mayor del esperado, fue el riego para el control de partículas en suspensión, cuyo valor teórico fue $3.936 .000 \mathrm{~m}^{3}$ y el consumo real de $6.010 .000 \mathrm{~m}^{3}$, es decir, un $53 \%$ mayor al previsto. Teniendo en cuenta los resultados alcanzados en este estudio, es posible estimar que en el rubro de la construcción hay un consumo de agua bastante superior a la demanda teórica. En este sentido, se hace necesario el desarrollo de estudios detallados basados en consumos reales que garanticen la sustentabilidad de este recurso hídrico en zonas áridas.

Palabras clave: Pérdidas de agua, indicadores de consumo de agua, construcción, sustentabilidad

\section{ABSTRACT}

In recent years, water scarcity in northern Chile has increased markedly, leading to an over exploitation of groundwater aquifers. In the construction sector, water is used in large quantities throughout the different activities of the construction projects, this fact makes essential to quantify the water consumption in order to know the real demand for water, and thus to have a clear and precise vision about the volumes of this resource which are used in the different activities of a construction project. In this study, the water consumption coefficients in relation to the different items corresponding to the construction of 219 homes and 2 multipurpose rooms were determined. For this purpose, the theoretical results of water demand obtained by 
means of calculations of the project, were compared with the results obtained in the real consumption of water in-situ. Results obtained for theoretical and real consumption of the tasks showed that the theoretical water volume to be used in the work was $10,946 \mathrm{~m}^{3}$, this value differs with the real volume of $16,283 \mathrm{~m}^{3}$ of water used in the project, i.e., it was used an additional volume of $5,337 \mathrm{~m}^{3}$. The latter implies an increase of $48.8 \%$ in water consumption, if compared to the theoretical water volume. The main water consuming activities were the following: soil compaction, irrigation to control particles in suspension, curing of concretes and mortars, worker camp, manufacture of concrete and mortars. Within these activities, the one that presented a higher than expected consumption was the irrigation for the control of suspended particles, whose theoretical value was $3,936,000 \mathrm{~m}^{3}$ and the real consumption was $6,010,000 \mathrm{~m}^{3}$, that is $53 \%$ higher than the provided. Considering the results obtained in this study, it is possible to estimate that in the construction sector there is higher water consumption, in comparison with the theoretical demand. In this sense, it is necessary to develop detailed studies based in the actual water consumption that guarantee the sustainability of this resource in arid zones.

Keywords: Water losses, water consumption indicators, construction, sustainability

\section{INTRODUCCIÓN}

No quedan dudas de la importancia que el agua tiene para la vida en el planeta tierra, sin embargo, a menudo se provocan pérdidas en la cantidad y calidad de las reservas de agua por las actividades antropogénicas. La Dirección General de Aguas [1] afirma que Chile en su conjunto, es considerado un país privilegiado en materia de recursos hídricos. El volumen de agua procedente de las precipitaciones que escurren por los cauces superficiales y subterráneos equivale a un promedio nacional per cápita de $51.218 \mathrm{~m}^{3} /$ persona/año, valor bastante más alto que la media mundial de $6.600 \mathrm{~m}^{3} /$ persona/año y muy superior al valor de 2.000 $\mathrm{m}^{3} /$ persona/año considerado internacionalmente como el umbral para el desarrollo sostenible [1]. Sin embargo, cuando se analiza regionalmente este valor promedio, se hace evidente la heterogeneidad hídrica del país. En el norte, prevalecen condiciones de escasez, donde la escorrentía per cápita promedio está por debajo de $\operatorname{los} 500 \mathrm{~m}^{3} /$ persona/año, mientras que las regiones del centro y sur del país, superan los $7.000 \mathrm{~m}^{3} /$ persona/año, llegando a un valor de $2.950 .168 \mathrm{~m}^{3} /$ persona/año en el extremo Sur [1]. A modo de ejemplo, en el período 2008-2016, considerando la compleja situación que vivió la Región de Atacama, producto de la prolongada sequía que afecta a la zona norte y centro de Chile, el Ministerio de Obras Públicas (MOP) decretó Zona de Escasez Hídrica en diferentes comunas [2].

El gran desafío propuesto por las autoridades del Gobierno de Chile, está enfocado en disminuir el impacto que está ocasionando o puede ocasionar la sequía a fin de permitir la sostenibilidad del recurso a lo largo del tiempo [1]. Con el propósito de velar por el uso eficiente del agua en zonas áridas del país, el Gobierno ha propuesto las Estrategias de Desarrollo Regionales para la macro zona norte de Chile, con políticas que garanticen el uso eficiente y sustentable del recurso hídrico, así como también, desarrollar una cultura ciudadana empresarial y productiva relativa al uso responsable del recurso, e incorporar alternativas para su suministro y gestión $[3,4,5,6]$.

En el norte chileno, la minería y la agricultura demandan ingentes cantidades de agua dulce para sus procesos productivos, sin embargo, en otros sectores como la construcción, el agua es un recurso que está presente de manera importante ya que se utiliza en diferentes partidas de una obra: en la compactación de suelos, como agente neutralizador de partículas en suspensión, en la mezcla y curado de hormigón, en mezclas adhesivas de revestimientos, en el lavado de agregados pétreos, en la limpieza de moldes y en las pruebas de presión, entre otras [7]. Al respecto, se hace necesario proponer estrategias que apunten a la eficiencia en el uso del agua en las obras de construcción.

Actualmente se reportan algunos estudios sobre la cantidad de agua utilizada en una obra de construcción para diferentes actividades y procesos. Aunque no hay consenso en las unidades de medida del consumo de agua en una obra de construcción, algunos autores $[8,9,10]$ utilizan el volumen de agua referido a las horas hombre $\left(\mathrm{m}^{3} / \mathrm{Hh}\right)$, volumen mensual $\left(\mathrm{m}^{3} / \mathrm{mes}\right)$ y al volumen sobre área construida $\left(\mathrm{m}^{3} / \mathrm{m}^{2}\right)$. Las investigaciones principalmente se focalizan en el consumo de agua potable en construcciones residenciales y comerciales. Además, la literatura aporta estudios de consumo de agua dulce en construcciones de países que tienen agua en abundancia, sin embargo, es escasa la información en zonas áridas y semi áridas. La información de estosestudios muestra resultados no concluyentes debido a su variabilidad, la que depende de varios factores, entre los que se pueden mencionar, el sistema constructivo, tamaño de la obra, tipo de obra, hábitos de uso del recurso agua por los trabajadores, entre otros. COSTA FILHO et al. [8] presentó datos del consumo de agua potable en 7 obras de construcción, cinco proyectos de departamentos residenciales y dos comerciales en Recife, Brasil, los que fueron monitoreados durante un año. El volumen de consumo de agua mensual fue 
variable, aún teniendo presente que las características de las obras de edificación eran similares. Los resultados indicaron un rango de consumo de agua entre $0,01-0,03 \mathrm{~m}^{3} / \mathrm{Hh}$ y entre $100-400 \mathrm{~m}^{3} / \mathrm{mes}$, siendo las principales actividades consumidoras de agua: el hormigón, morteros, curado del hormigón, control del polvo, pruebas de impermeabilización, pinturas al látex, limpieza final de la obra y de las fachadas. Además, DUBRAVIC [9] estudió el consumo de agua en una vivienda tipo de dos plantas en Bolivia, con una superficie de 100 a $200 \mathrm{~m}^{2}$, llegando a establecer que el consumo de agua fue de $0,684 \mathrm{~m}^{3} / \mathrm{m}^{2}$, donde las actividades consumidoras fueron: curado de hormigones, morteros y revoques, riego de encofrados, aditivos (62\%), pinturas al agua, proceso de compactación (23\%), herramientas y equipos (14\%); elaboración y curado de probetas, impermeabilidad de ventanas y cubierta (1\%). También, MARQUES et al. [10] estudiaron el consumo de agua y energía de 6 edificios de departamentos de una constructora en Passo Fundo en Brasil, indicando que el consumo de agua estaba comprendido en el rango $0,01-0,28 \mathrm{~m}^{3} / \mathrm{m}^{2}$, donde las principales actividades consumidoras de agua fueron: fugas en tuberías, pérdidas en grifos y llaves, pérdidas en baños sanitarios, mezclas para hormigón y morteros, curado de hormigones. PESSARELLO [11] estudió el consumo de agua de tres edificios de departamentos (lujo, medio, normal) obteniendo coeficientes de $0,68 \mathrm{~m}^{3} / \mathrm{m}^{2}(0,0144$ $\left.\mathrm{m}^{3} / \mathrm{Hh}\right), 0,44 \mathrm{~m}^{3} / \mathrm{m}^{2}\left(0,0099 \mathrm{~m}^{3} / \mathrm{Hh}\right)$ y $0,37 \mathrm{~m}^{3} / \mathrm{m}^{2}\left(0.0116 \mathrm{~m}^{3} / \mathrm{Hh}\right)$, respectivamente. El autor indica que la variación del consumo de agua en las diferentes obras, se debe al tamaño del edificio, a las características técnicas utilizadas y a su complejidad. En la Tabla 1 se presenta el consumo de agua potable durante la ejecución para diferentes obras de construcción.

Respecto de la metodología de levantamiento de datos del consumo de agua en las obras de construcción utilizada por otros investigadores, se puede señalar la instalación de un medidor de consumo de agua en el sector de vestuario, con el propósito de cuantificar el volumen de agua utilizada por los trabajadores [12]. Por otra parte, COSTA FILHO et al. [8] consideraron el consumo de agua potable, número de empleados, costo del agua potable y fases de la obra, así como el monitoreo de consumo de agua de las actividades de la administración de la obra, reutilización y desperdicio del agua. Otros investigadores [14], identificaron las fuentes de consumo a través de visitas técnicas y entrevistas realizadas a los empleados, y por otra parte, estimaron el consumo de agua de la obra a partir de indicadores de obras similares. En el presente estudio, los datos de consumo de agua se obtuvieron de dos fuentes diferentes: la primera, de una fuente de abastecimiento directa, la cual correspondió a la información registrada en las facturas de consumo de la empresa distribuidora de agua potable local registrada en el medidor instalado en la obra; la segunda fuente, indirecta, correspondió al registro del agua transportada en camiones aljibes de un proveedor externo, para su utilización en el control de partículas en suspensión y además, al registro del agua utilizada en la preparación del hormigón premezclado abastecido por proveedores externos.

Tabla 1: Coeficientes de consumo de agua potable en obras de construcción obtenidos por diferentes autores.

\begin{tabular}{l|l|l|l}
\hline TIPO DE OBRA & ÁREA CONSTRUIDA, $\mathbf{~ m}^{\mathbf{2}}$ & CONSUMO DE AGUA, $\mathbf{~}^{\mathbf{3}} \mathbf{m}^{\mathbf{2}}$ & REFERENCIA $^{\mathbf{2}}$ \\
\hline Residencial & 6.624 & 0,28 & {$[10]$} \\
\hline $\begin{array}{l}\text { Residencial } \\
\text { Comercial }\end{array}$ & 15.969 & 0,01 & {$[10]$} \\
\hline Residencial & 13.705 & 0,17 & {$[10]$} \\
\hline Residencial & 5.568 & 0,18 & {$[10]$} \\
\hline Residencial & 5.046 & 0,13 & {$[10]$} \\
\hline Comercial & 48.871 & 0,02 & {$[10]$} \\
\hline Residencial & 7.468 & 0,83 & {$[12]$} \\
\hline Residencial & 120.000 & 0,68 & {$[11]$} \\
\hline Residencial & 5.100 & 0,44 & {$[11]$} \\
\hline Residencial & 4.200 & 0,17 & {$[11]$} \\
\hline Vivienda & $100-200$ & 0,68 & {$[9]$} \\
\hline Comercial & 2.682 & 0,23 & {$[13]$} \\
\hline Comercial & 15.210 & 0,20 & {$[13]$} \\
\hline Comercial & 3.843 & 0,25 & {$[13]$} \\
\hline
\end{tabular}

Dada la importancia que tiene el recurso hídrico en la construcción, el objetivo de este estudio fue identificar el gasto de agua potable, las principales actividades consumidoras de agua y las fuentes de pérdidas en una obra de construcción residencial de 219 viviendas y dos salas multiuso emplazada en la ciudad de Copiapó, en una zona semi árida situada en la Región de Atacama, en el norte de Chile. 


\section{MATERIALES Y MÉTODOS}

\subsection{Caracterización del proyecto estudiado}

El estudio consideró el consumo de agua durante la fase de construcción de un proyecto de 219 viviendas unifamiliares y dos salas multiusos, situado en Av. Parque Los Carrera Norte $\mathrm{s} / \mathrm{n}^{\circ}$, Copiapó, Chile. La constructora forma parte de un consorcio dedicado a la edificación, con una experiencia de 35 años en el rubro y posicionada como líder en el mercado inmobiliario nacional. Las viviendas unifamiliares son de dos tipos, distribuidas en dos plantas, con una superficie total de $11.800 \mathrm{~m}^{2}$. Las viviendas Tipo A tienen superficies de $52,10 \mathrm{~m}^{2}$ (pareada) y 53,00 $\mathrm{m}^{2}$ (aislada), y las Tipo B, tienen superficies 63,63 $\mathrm{m}^{2}$ (pareada) y 64,49 $\mathrm{m}^{2}$ (aislada). Por otra parte, las salas multiuso presentan superficies de $100,84 \mathrm{~m}^{2}$ y $80,10 \mathrm{~m}^{2}$, ambas distribuidas en una planta.

\subsection{Obtención de los datos}

Primeramente se realizó un cálculo teórico a partir de la información contenida en los planos y especificaciones técnicas del proyecto. Este procedimiento permitió cuantificar el volumen de agua necesario para cada partida de la obra. Posteriormente, se determinó el consumo real de la obra. Para ello, se identificaron dos fuentes de ingreso de agua al sistema constructivo, una de ingreso directo, que incluyó el abastecimiento de la planta de agua de la ciudad, y fuente de ingreso de agua indirecta, que corresponde al abastecimiento desde fuentes externas, tales como camiones aljibe necesario para el control de polvo en suspensión, y agua de los hormigones premezclados fabricados por un contratista externo.

En el desarrollo de este estudio, se realizó un seguimiento en terreno a todos los procesos constructivos de la obra, identificándose las siguientes actividades en las que se utilizó agua potable: compactación de suelos con material estabilizado, riego de caminos para evitar partículas en suspensión, mezclas de hormigón realizadas por la empresa constructora, mezclas de hormigón de los proveedores externos, curado del hormigón, lavado de los materiales pétreos, lavado de los moldes de losas, pruebas de presión de redes de agua y alcantarillado, agua utilizada en el campamento para usos sanitarios y domésticos, y preparación de mezclas de adhesivos.

Para conocer el volumen de agua utilizado en la obra, se siguió el siguiente procedimiento: a) Para el agua ingresada en forma directa, se tomaron los datos de lectura del medidor instalado en la obra por el único proveedor de agua potable de la ciudad; b) el agua utilizada en forma indirecta, se obtuvo a partir del volumen total de agua potable abastecida por los camiones aljibes para el riego del control de partículas en suspensión y compactación de suelos, durante un período de 8 meses; y a partir de los datos entregados por el proveedor de hormigón premezclado. En la Figura 1 se muestran las principales partidas consumidoras de agua potable de la obra de construcción estudiada. 
tilización de agua en la construcción

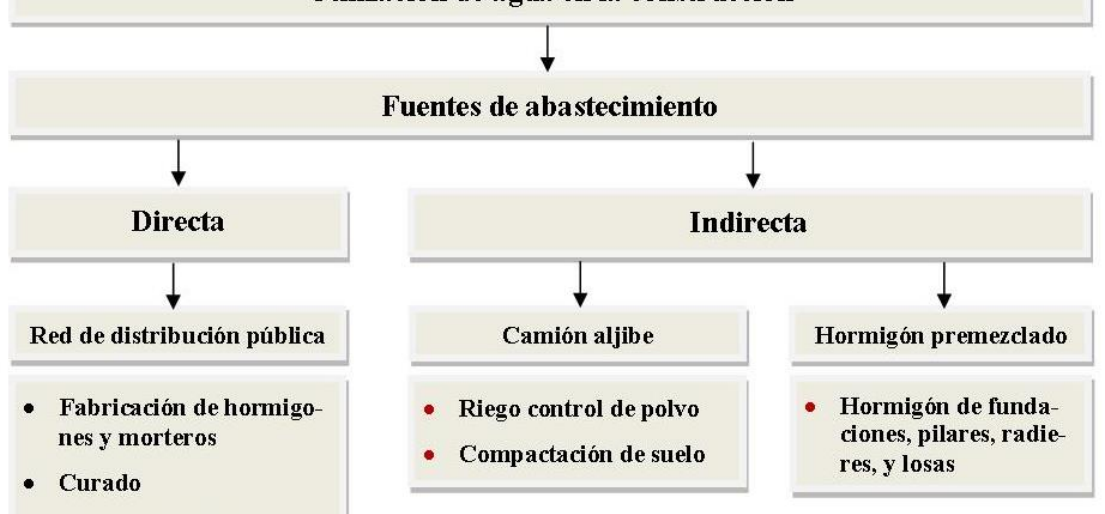

- Lavado de árido

- Lavado de moldajes

- Pruebas de presión red de agua y alcantarillado

- Mezclas en adhesivos

- Agua utilizada en campamento

Figura 1: Principales actividades consumidoras de agua en la obra de construcción de viviendas estudiadas.

Para el cálculo teórico del consumo de agua de cada proceso constructivo, se contó con la información y la autorización de la empresa constructora, respecto del estudio de los siguientes antecedentes de la obra: planos de cada tipo de vivienda; planos de las salas de multiuso; planos de alcantarillados y agua potable públicos; planos de pavimentación y áreas verdes; dosificaciones utilizadas en la planta de hormigón; informes de análisis de laboratorio de hormigones y suelos, entre otros; cantidad de trabajadores que alojaron en el campamento de la obra; y la autorización para el acceso total a la obra en construcción.

\subsection{Análisis de los datos}

Para la obtención de los datos del consumo de agua, se comparó el gasto teórico con el gasto real medido in situ durante el periodo de construcción. Para determinar el gasto real de agua por partida, la cuantificación del consumo de agua de la fuente externa se realizó mediante las lecturas del medidor general de agua que se encontraba en la obra. Por otra parte, el consumo de agua de los camiones aljibe, se determinó en función de la capacidad de almacenamiento de cada camión que ingresaba a la obra, registrándose la fecha y volumen de agua ingresada a la obra durante el período de ejecución de la obra de construcción. Respecto del volumen de agua utilizada en la fabricación de los hormigones premezclados, el proveedor entregó la información basada en las dosificaciones utilizadas para cada tipo de hormigón. Por otra parte, durante las diferentes etapas constructivas, se realizaron inspecciones y monitoreo in situ para determinar las fuentes de pérdidas de agua y sus causas.

Para el caso de la determinación del gasto teórico de agua, el levantamiento de datos por partida se realizó de acuerdo al procedimiento que se detalla a continuación. Para obtener el agua en el proceso de compactación de suelos con material estabilizado, se estudiaron los certificados del ensayo Proctor Modificado de un laboratorio de mecánica de suelos externo a la constructora, documentos que aportaron la información de la humedad óptima requerida en las actividades de la compactación del suelo. Posteriormente, estos datos fueron corroborados en terreno mediante pruebas de humedad y densidad del suelo compactado utilizando un densímetro nuclear. Para el cálculo teórico del agua de riego utilizada para neutralizar las partículas en suspensión de los caminos, se estudiaron las especificaciones técnicas y cubicaciones del proyecto que indicaron una superficie total de $4,578 \mathrm{~m}^{2}$. Para ello, se estimó el aporte de agua diario considerando un camión con $16 \mathrm{~m}^{3}$ de capacidad y con un tiempo de descarga de 30 minutos, lo que permite descargas 3.48 $\mathrm{L} / \mathrm{m}^{2}$. Sin embargo, durante la ejecución de la obra, se contrataron los servicios de provisión de agua con camiones de $12,13,15$ y $16 \mathrm{~m}^{3}$, con tiempo de descarga comprendido en el rango de 20 a 30 minutos. El cálculo de las mezclas de hormigón realizadas tanto en la obra como por los proveedores externos, se realizó 
utilizando los planos que se manejaban en la obra, lo que permitió realizar las cubicaciones respectivas y determinar los volúmenes de hormigón que se necesitaban en cada partida y para cada tipo de vivienda. Reunida esta información, se procedió a calcular la cantidad de agua que debía ser ocupada en todo proceso constructivo. En relación al agua requerida para el curado del hormigón, este proceso debía realizarse tres veces al día a todos los hormigones, excepto a las partidas emplantillados, radieres, losas y veredas, los cuales fueron curados con una membrana impermeable. Para el cálculo teórico se realizaron pruebas de curado y se tomó el tiempo de demora, repitiendo las pruebas seis veces por vivienda y por partida. En la determinación del agua para mezclas de adhesivo cerámico, yeso, fragüe y masilla base, para el cálculo teórico del agua que se debía ocupar en la obra se tomaron en cuenta las dosificaciones de agua recomendadas por los proveedores. En la determinación de la cantidad real de agua utilizada en el lavado de los materiales pétreos constituyentes del hormigón, se realizó una prueba que consistió en medir el tiempo que se requería para la limpieza de los áridos, registrando el volumen de agua consumida en la actividad. En el proceso de lavado de los moldajes de losas, para la determinación de la cantidad de agua cercana a lo real, se realizó una prueba similar a la anteriormente explicada. Respecto del agua utilizada en las pruebas de presión de redes de agua y alcantarillado, se registró el volumen de agua que ingresaba en cada uno de los sistemas de tuberías de la obra. En el caso del agua utilizada en el campamento para usos sanitarios y domésticos, el cálculo teórico se determinó a partir de la cantidad de trabajadores que pernoctaron en el campamento, los días trabajados, estimando una cantidad de $150 \mathrm{~L} /$ habitante/día, esta cantidad se obtuvo de valores establecidos en ingeniería sanitaria, lo que oscilan entre 80 a 450 L/día.

\section{RESULTADOS}

El resultado del consumo teórico de agua de las principales partidas, obtenido por cálculo del consumo esperado de agua del proyecto, se resume en la Tabla 2. En ella se muestra la relación porcentual del gasto de cada partida referido al consumo total de agua en la obra de construcción. Se puede observar que el mayor consumo calculado corresponde a la partida riego para el control de partículas en suspensión con un $36 \%$ respecto del total, seguido del agua de curado de morteros y hormigones con $26 \%$.

Tabla 2: Gasto teórico de agua.

\begin{tabular}{l|l|l}
\hline PARTIDA & GASTO TEÓRICO, $\mathbf{~}^{3}$ & GASTO TEÓRICO/GASTO TOTAL, \% \\
\hline Compactación del suelo & $1.543,038$ & 14,1 \\
\hline $\begin{array}{l}\text { Riego para control de partículas en suspen- } \\
\text { sión }\end{array}$ & $3.936,000$ & 36,0 \\
\hline Lavado de áridos hormigón & 16,062 & 0,2 \\
\hline Fabricación hormigones y morteros & 572,175 & 5,2 \\
\hline Curado hormigones y morteros & $2.835,376$ & 25,9 \\
\hline Agua en mezclas adhesivos & 34,450 & 0,3 \\
\hline Limpieza moldajes losas & 14,580 & 0,1 \\
\hline Pruebas presión alcantarillado & 69,197 & 0,6 \\
\hline Pruebas presión red agua potable & 12,995 & 0,1 \\
\hline Campamento trabajadores & $1.912,650$ & 17,5 \\
\hline TOTAL & $10.946,523$ & 100,0 \\
\hline
\end{tabular}

En Tabla 3, se muestra el consumo teórico correspondiente al agua que ingresa directamente a la obra desde la matriz de agua potable, el gasto por el riego con camiones aljibe para control de partículas en suspensión y el agua contenida en los hormigones fabricados en la obra. Además, se muestra la relación porcentual del consumo teórico de cada partida referido al consumo teórico total de agua en la obra. El consumo directo de agua incluye los siguientes partidas: compactación del suelo, lavado de áridos de hormigones, fabricación y curado de hormigones y morteros, agua necesaria para las mezclas adhesivas, limpieza de moldes de hormigón de losas, pruebas de presión de alcantarillado y agua potable, y el consumo de agua del campamento de trabajadores. Por otra parte, se aprecia que los mayores consumos de la obra corresponden al agua que ingresa en forma directa desde la matriz pública a la red interior con un $64 \%$, seguido del agua para el riego del control de partículas en suspensión con $36 \%$. 
Tabla 3: Gasto teórico directo e indirecto de agua.

\begin{tabular}{l|l|l}
\hline PARTIDA & GASTO TEÓRICO, $\mathbf{~ m}^{3}$ & GASTO TEÓRICO/GASTO TOTAL, \% \\
\hline Consumo directo agua obra & $7.010,523$ & 64,0 \\
\hline $\begin{array}{l}\text { Riego para control partículas en sus- } \\
\text { pensión }\end{array}$ & $3.936,000$ & 36,0 \\
\hline TOTAL & $10.946,523$ & 100,0 \\
\hline
\end{tabular}

En Tabla 4 se muestra el consumo real de agua directa e indirecta en la obra. En ella se observa que el mayor consumo corresponde al agua que ingresa en forma directa a la obra de construcción.

Tabla 4: Gasto real directo e indirecto de agua.

\begin{tabular}{l|l|l}
\hline PARTIDA & GASTO REAL, $\mathbf{~}^{3}$ & GASTO REAL/GASTO TOTAL, $\%$ \\
\hline $\begin{array}{l}\text { Agua registrada en contador de la } \\
\text { obra }\end{array}$ & $9.843,000$ & 60,4 \\
\hline $\begin{array}{l}\text { Riego para el control de parículas en } \\
\text { suspensión }\end{array}$ & $6.010,000$ & 36,9 \\
\hline Hormigón premezclado & 433,341 & 2,7 \\
\hline TOTAL & $16.286,341$ & 100,0 \\
\hline
\end{tabular}

Si se comparan los resultados de Tabla 3 y Tabla 4, se observa que los consumos directos e indirectos de agua del gasto teórico y gasto real representan porcentajes con respecto al gasto total muy similares, sin embargo debe tenerse presente que el cálculo en volumen deja de manifiesto que existe un incremento notorio en el volumen consumido de agua real respecto del cálculo teórico. Para el caso del consumo directo de agua este incremento es de 40,4\%, mientras que el consumo indirecto de agua correspondiente al riego para control de partículas en suspensión este incremento fue de un 52,7 \%. Debe tenerse presente que el consumo real de agua en la fabricación hormigones y morteros, corresponde a una disminución de un $24,3 \%$ respecto del teórico.

Durante el estudio, se observó un consumo excesivo de agua para el control de las partículas en suspensión, así como pérdidas de agua en tuberías y mangueras rotas, grifos defectuosos, roturas de tuberías subterráneas causadas por retroexcavadoras, riego innecesario de radieres con membranas de curado y grifos y tuberías mal cerradas, las que permitían un flujo de agua sin control lo que se traduce en un aumento de los costos reales de una obra de construcción con el consiguiente desperdicio de un recurso natural escaso en la Región de Atacama.

En relación al consumo de agua real al término de la obra de construcción estudiada, se obtuvo un valor de $1,38 \mathrm{~m}^{3} / \mathrm{m}^{2}$, mientras que el indicador teórico fue de $0,93 \mathrm{~m}^{3} / \mathrm{m}^{2}$ (Tabla 5).

Tabla 5: Consumo real y teórico de agua en $\mathrm{m}^{3} / \mathrm{m}^{2}$.

\begin{tabular}{l|l|l}
\hline PARTIDA & CONSUMO TEÓRICO, $\mathrm{m}^{3} / \mathrm{m}^{2}$ & CONSUMO REAL, $\mathrm{m}^{3} / \mathrm{m}^{2}$ \\
\hline Consumo directo agua obra & 0,594 & 0,834 \\
\hline $\begin{array}{l}\text { Riego para el control de partículas en } \\
\text { suspensión }\end{array}$ & 0,334 & 0,509 \\
\hline Hormigón premezclado* & 0,000 & 0,037 \\
\hline TOTAL & 0,928 & 1,380 \\
\hline
\end{tabular}

* El hormigón premezclado corresponde a un fabricante externo a la empresa constructora.

\section{DISCUSIÓN}

Como puede observarse en la Tabla 1 los valores usuales de consumo de agua en construcciones de obras residenciales, principalmente ejecutadas en Brasil, país con superhábit del recurso hídrico, oscilan entre $0,01 \mathrm{~m}^{3} / \mathrm{m}^{2}$ [9] a $0,83 \mathrm{~m}^{3} / \mathrm{m}^{2}$ [12]. Sin embargo los resultados obtenidos en el estudio realizado en Atacama, entregó un coeficiente de $1,38 \mathrm{~m}^{3} / \mathrm{m}^{2}$, siendo un $66,3 \%$ superior al valor obtenido por DOS SANTOS et al. [12]. Teniendo en cuenta que la región de Atacama es una zona de escasez hídrica, resulta alarmante el consumo de agua obtenido en la construcción estudiada. Las principales causas que generan este alto consumo, se deben por una parte, al excesivo riego para el control de material particulado en suspensión de la obra, principalmente en el período 
estival, y por otra, a las pérdidas detectadas en grifos, tuberías y a la falta de conciencia en el ahorro del recurso hídrico por parte de los trabajadores implicados en la construcción. En este sentido, en la literatura se han reportado como principales actividades consumidoras de agua, la mezcla para hormigones y morteros, el curado del hormigón, el control del polvo, la limpieza final de la obra y de las fachadas [8], y también se ha indicado que el consumo mayor de agua en las obras analizadas ocurría en las etapas de terminaciones, la que involucraba todos los usos en limpieza, preparación de materiales y consumo propio [14].

Respecto de la forma como se tomaron los datos en las obras de construcción de Brasil, DOS SANTOS et al. [12] , investigaron el consumo de agua en un sitio de construcción en la ciudad de Recife/PE, para ello seleccionaron un edificio residencial de alto estándar, ubicado en un barrio de la ciudad, el que constaba de 17 pisos y una área construida total de 7.467,66 $\mathrm{m}^{2}$. En este trabajo se instalaron contadores de agua en diferentes sectores de la obra e instalando otro contador en el vestuario, con el objetivo de observar el comportamiento del agua utilizada en este entorno, ya que se especula que los mayores consumidores de agua en un sitio de construcción son los empleados. COSTA FILHO et al. [8] realizaron un análisis de los registros mensuales de las siguientes variables: consumo de agua potable, número de empleados, costo del agua potable y fases de la obra. Estos investigadores seleccionaron siete obras de una constructora las que fueron monitoreadas durante el año 2012, siendo observadas las actividades que involucraban la administración, la reutilización y el desperdicio del agua. Por otra parte, NAPOMUCENO E DA PAZ [14] para la identificación de las fuentes de consumo en el sitio de obra del IFPE en el campus Cabo de San Agustín del Estado de Pernambuco, realizaron un levantamiento de las fuentes de la obra, para ello la identificación de las fuentes se realizó con visitas técnicas y mediante entrevistas a los empleados. Para la estimación del consumo de agua de la obra a partir de indicadores de otras obras, los datos analizados proceden de la base de datos del grupo de investigación, proveniente de otras investigaciones realizadas anteriormente. En el presente estudio realizado en Chile, los datos de consumo de agua de la obra fueron obtenidos de la información registrada en las siguientes fuentes de consumo: agua ingresada directamente a la obra, cuyos datos se registraban en un contador instalado por la empresa proveedora externa; agua utilizada en forma indirecta, correspondiente a camiones aljibes de un proveedor externo, el recurso hídrico se utilizaba en el riego para el control de partículas en suspensión; agua utilizada en forma indirecta para la preparación de hormigón premezclado, servicio ejecutado por proveedores externos.

Diversas medidas pueden proponerse para minimizar el consumo de agua en el proceso constructivo, que van desde que el diseño arquitectónico considere materiales que demanden bajo consumo de agua, hasta ciclos educativos dirigido a los trabajadores de la construcción. Es importante además, determinar los focos de pérdida de agua durante la ejecución de la obra, a través de un monitoreo constante y sistemático. Las fuentes de pérdida de agua radican en cañerías rotas, estanque acumuladores con fugas, grifos en mal estado, infiltraciones, entre otras. En la Figura 2, se observan charcos originados por pérdidas de agua debido a grifos abiertos sin control y a roturas de mangueras.
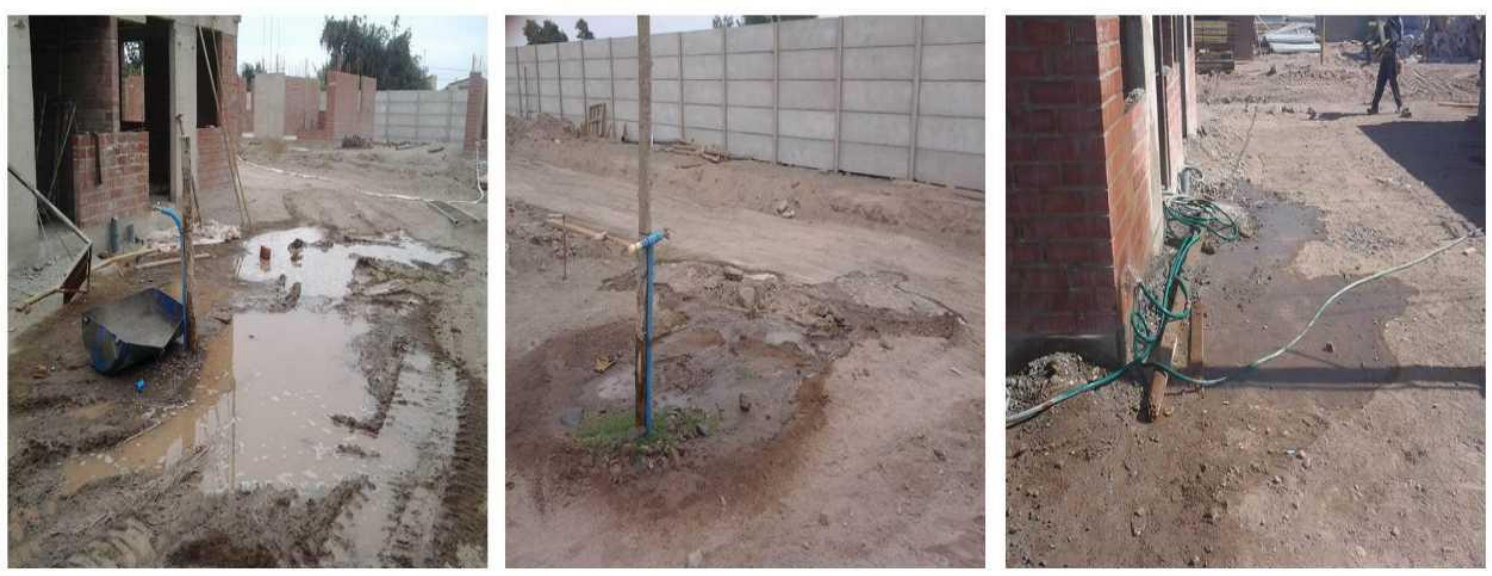

Figura 2: Pérdidas de agua en grifos y mangueras.

Además, es interesante poder establecer algunas medidas que apunten a la eficiencia del uso del agua potable en la obra de construcción, tales como afiches ilustrativos y charlas motivadoras de concientización de los trabajadores, uso de contadores de consumo de agua por sectores en el lugar de la obra, uso de grifos con difusores y accionamiento automático, utilización de fuentes alternativas de agua potable (sistemas atrapanieblas, 
reúso de aguas de rechazo de procesos de osmosis inversa, reúso de agua grises para alimentar urinarios, entre otros), uso de productos industriales líquidos para impermeabilizar los hormigones evitando así, el curado por riego. En la literatura también se sugieren acciones para disminuir el consumo de agua en las obras de construcción, PESSARELLO [11] recomienda la utilización de grifos con accionamiento o cierre automático, la instalación de temporizadores en las duchas para limitar el tiempo de baño, el uso de fuentes alternativas de agua, charlas para la concientización de los funcionarios, y seguimiento mensual de los consumos de agua y de las medidas aplicadas. Por otra parte, COSTA FILHO et al. [8] sugieren utilizar dispositivos en la salida de las llaves para disminuir el paso del agua y también para transformar el chorro de agua en pequeños chorros, válvulas para reducir el consumo en los urinarios de los baños, pistola de riego de chorro ajustable para las mangueras, y empleo de sacos de tejidos en el proceso de curado del hormigón.

Respecto al beneficio económico que significaría para la empresa constructora el ahorro del agua consumida en exceso, si se considera que en Copiapó en abril/2018 el agua tenía un costo de $1.860 \$ / \mathrm{m}^{3}$ (USD $3 / \mathrm{m}^{3}$ ) y el consumo en exceso de agua fue $5.340 \mathrm{~m}^{3}$, esto significaría un valor total de $\$ 9.932 .400$ (USD 16.020). Al parecer el beneficio económico no resulta tan significativo para el tamaño de la obra, sin embargo, si se considera que las viviendas construidas están ubicadas en una zona semi-árida donde este recurso hídrico no es abundante, el consumo excesivo de agua es un llamado de alerta para los empresarios constructores, en relación a la necesidad de realizar una mejor gestión de este recurso en las obras, evitando su desperdicio y consumo exagerado. En este sentido, las medidas propuestas en este trabajo para minimizar el consumo de agua en el proceso constructivo, son una contribución positiva para la solución de este problema.

\section{CONCLUSIONES}

Se estudió el consumo de agua potable en la construcción de 219 viviendas y dos salas multiuso en una ciudad ubicada en el Desierto de Atacama. Los resultados indicaron un consumo real de $1,38 \mathrm{~m}^{3} / \mathrm{m}^{2}$, cantidad que es mayor en un $66,3 \%$ que el máximo valor obtenido en una edificación de edificio de departamentos en Brasil. Las principales fuentes de pérdidas de agua potable en la obra estudiada fueron tuberías rotas, riego excesivo para el control de material particulado en suspensión de la obra, poco cuidado en el cierre de grifos por parte de los trabajadores. Para la construcción en zonas áridas se hace necesario adoptar medidas que mitiguen las pérdidas de agua, considerando el control dirigido al uso eficiente del agua en cada etapa de la obra y proceso educativo permanente de los trabajadores.

La falta de control en el consumo de agua en una construcción, se traduce en un aumento de los costos reales de la obra, con el consiguiente desperdicio de un recurso natural escaso en una zona semi-desértica, situación que agrava la escasez de agua en la Región de Atacama comprometiendo el manejo sustentable de este recurso estratégico para el desarrollo humano y económico de la zona.

\section{BIBLIOGRAFIA}

[1] DGA, MOP, CHILE, “Atlas del agua Chile 2016”, http://www.dga.cl/atlasdelagua/Paginas/default.aspx. Visitada en noviembre de 2017

[2] DGA, MOP, CHILE, "Decretos zonas de escasez hídrica", http://www.dga.cl/administracionrecursoshidricos/decretosZonasEscasez/Paginas/default.aspx. Visitada en octubre de 2017.

[3] GOBIERNO REGIONAL ATACAMA, "Estrategia Regional de Desarrollo de Atacama 2007-2017", http://goreatacama.gob.cl/estrategia-regional-de-desarrollo-de-atacama-2007-2017/. Visitada en octubre de 2017.

[4] SUBDERE, "Antofagasta-Estrategia Regional de Desarrollo Periodo 2009-2020", http://www.subdere.gov.cl/documentacion/antofagasta-estrategia-regional-de-desarrollo-periodo-2009-2020. Visita en noviembre de 2017.

[5] SUBDERE, "Tarapacá-Estrategia Regional de Desarrollo Periodo 2011-2020", http://www.subdere.gov.cl/documentacion/tarapaca-estrategia-regional-de-desarrollo-periodo-2011-2020. Visitada en noviembre de 2017.

[6] SUBDERE, "Coquimbo-Estrategia Regional de Desarrollo Periodo 2009-2020", http://www.subdere.gov.cl/documentacion/coquimbo-estrategia-regional-de-desarrollo-periodo-2009-2020. Visitada en octubre de 2017.

[7] ZUÑIGA, B., Identificación y cuantificación del consumo de agua en la construcción de la obra Las Brisas segunda etapa, Proyecto de Título de Constructor Civil y Grado de Licenciado en Construcción Civil, Instituto Tecnológico de la Universidad de Atacama, Copiapó, Chile, 2015. 
[8] COSTA FILHO, E., DA SILVA, S.R., BRITO, I.G.G., "Consumo de agua em canteiros de obra da Região Metropolitana do, Recife”, In: Encontro Latinoamericano de Edificações e Comunidades Sustentáveis, Curitiba, 21-24 Outubro 2013.

[9] DUBRAVIC, A., "Quantification of wáter consumption during the construction process of single family housing type", In: Proceedings of the 3rd International Congress on Sustainable Construction and EcoEfficient Solutions, pp. 1084-1107, Sevilla, España, March 2017.

[10] MARQUES, C.T., GOMES, B.M.F., BRANDLI, L.L., "Consumo de agua e energia em canteiros de obra: um estudo de caso do diagnóstico a ações visando a sustentabilidade", Ambiente Construído, v. 17, n. 4, pp. 79-90, Out. 2017.

[11] PESSARELLO, R.G., Estudo exploratorio quanto ao consumo de agua na produção de obras de edificios: avaliacão, Monografia do Título de Especialista em Tecnologia e Gestão da Producão de Edifícios, Escola Politécnicaa da Universidade de São Paulo, São Paulo,SP, Brasil, 2008.

[12] DOS SANTOS, C.P., DA SILVA, S.R., CERQUEIRA, C.A., "Water consumption in construction sites in the city of Recife/PE", EJGE, v. 20, pp. 1711-1726, 2015.

[13] DA SILVA, R.R. e TAKEDA, R.Y., “Gestão da agua em canteiros de obras de construcão civil”, In: VIII Encontro Internacional de Producão Científica, Maringá, Brasil, 22-25 Outubro 2013.

[14] NAPOMUCENO, S.C.B., PAZ, D.H.F., "Desenvolvimento de um programa de gestão da agua pro canteiro de obras de uma institução de ensino", In: VII Congreso Brasileiro de Gestão Ambiental, Campina Grande, PB, Brasil, 21-24 Novembro 2016. 